\title{
Fatores de risco e proteção na escola: Reprovação e expectativas de futuro de jovens paraenses
}

\author{
Tatiene Germano Reis Nunes \\ Universidade Federal do Pará - PA \\ Fernando Augusto Ramos Pontes \\ Universidade Federal do Pará - PA \\ Lucia Isabel da Conceição Silva \\ Universidade Federal do Pará - PA \\ Débora Dalbosco Dell'Aglio \\ Universidade Federal do Rio Grande do Sul - RS
}

\section{Resumo}

Este estudo investigou as relações entre reprovação escolar, percepções quanto à escola e expectativas de futuro entre jovens, a partir de dados de uma amostra de 610 jovens com idades entre 14 a 24 anos $(m=16,56 ; d p=2,37)$, de ambos os sexos, estudantes de escolas públicas da cidade de Belém do Pará. Foi utilizado um questionário com 77 questões de múltipla escolha. Os resultados revelaram que quase $50 \%$ dos alunos já sofreram reprovação escolar, embora apresentem boa percepção quanto à escola. Foram observadas correlações entre reprovação escolar e baixas expectativas de futuro acadêmico e entre boas percepções quanto à escola e melhores perspectivas acadêmicas. A expectativa de entrar na universidade apareceu mais associada ao sexo feminino. Os resultados poderão contribuir para o entendimento da importância da instituição escolar na promoção de fatores de proteção no desenvolvimento.

Palavras-chave: Jovens, fracasso escolar, expectativas.

\section{Risk and protective factors in school: Failure and future expectations of young in Pará}

\begin{abstract}
In this study we investigate the relations existing amid school failure, perceptions concerning the school and expectations of future among young people, from data of a sample of 610 young people with ages between 14 and 24 years $(m=16,56$; $d p=2,37$ ), of both sexes, students from public schools of Belém city in Pará state. A questionnaire with 77 multiple choice questions was used. The results revealed that almost half of students already presented school failure, although they present good perception concerning the school. Correlations between school disapproval and low expectations of academic future and between good perceptions concerning the school and best academic expectations were observed. The expectation of having access to the university appeared more associated to female sex. The results may contribute to the understanding of the importance of the school in promoting protective factors in the learners development.
\end{abstract}

Key words: Young adults, academic failure, expectations.

\section{Los Factores de riesgo y de protección en la escuela: reprobación y expectativas de futuro de jóvenes paraenses}

\section{Resumen}

Este estudio investigó las relaciones entre reprobación escolar, percepciones en cuanto a la escuela y expectativas de futuro entre jóvenes a partir de datos de uma muestra de 610 jóvenes con edades entre 14 y 24 años (promedio =16,56; sd=2,37), de ambos sexos, estudiantes de escuelas públicas de la ciudad de Belém do Pará. Se utilizó cuestionario con 77 preguntas de opción múltiple. Los resultados revelaron que casi la mitad de los alumnos ya han experimentado reprobación escolar, a pesar de que apresentan buena percepción de la escuela. Se observaron correlaciones entre reprobación escolar y bajas expectativas de futuro académico y entre buenas percepciones de la escuela y mejores perspectivas académicas. La expectativa de ingresar a la universidad está asociada más al sexo femenino. Los resultados pueden contribuir para el entendimiento de la importancia de la institución escolar en la promoción de factores de protección en el desarrollo.

Palabras Clave: juventud; reprobación; expectativa. 


\section{Introdução}

A educação, um direito institucional fundamental de todos os cidadãos (Constituição da República Federativa do Brasil, 1988), é da maior importância para o desenvolvimento humano e para a sociedade (Dessen \& Polonia, 2007; Gomes \& Horta, 2010; Tavares, 2002; Yunes \& Szymanski, 2001)). De acordo com o relatório da Unesco (1998), a educação, embora esteja ligada a diversos contextos e englobe variados formatos, necessita de adequação ainda no século $X X I$, especialmente no que se refere à educação escolar.

As dificuldades presentes no processo de educação escolar, em especial o desempenho precário e os problemas relacionais dos jovens, poderão comprometer 0 interesse pela escola. Além do mais, as sucessivas vivências de reprovações e de evasões podem levar ao abandono escolar. Assim, fenômenos que representam o fracasso escolar podem constituir-se como riscos ao desenvolvimento humano, levando a uma condição de vulnerabilidade social (Amparo, Galvão, Cardenas, \& Koller, 2008; Camargo \& Libório, 2010; Leon \& Menezes-Filho, 2002; MEC, 2005; UNESCO, 1998).

Embora estudos do IBGE (2013) demonstrem a democratização do acesso à escola, com um significativo aumento do percentual de alunos matriculados $(85,4 \%$ no Ensino Fundamental e 85,9\% no Ensino Médio em 2010), ainda pode ser observada certa falta de preparo da escola no que diz respeito à garantia da qualidade da aprendizagem e à promoção de um desenvolvimento positivo e de aprendizagem significativa para a vida e para o trabalho (MEC, 2005; Tacca \& Branco, 2008). Da mesma forma, Poletto e Koller (2008) demonstraram que na escola podem ser observados tanto os aspectos protetivos como de risco para o desenvolvimento humano. Apesar disso, estudos indicam a importância da instituição escolar para o desenvolvimento de crianças e de adolescentes nos seus planos de vida, incluindo expectativas de estudo e de trabalho, constituindo-se desta forma um fator de proteção (Bastos, 2005; Beutil, 2000; Camargo \& Libório, 2011).

\section{Fatores de risco e de proteção ao desenvolvimento}

Ao buscar compreender a realidade da juventude e sua relação com o ambiente escolar, é importante reconhecer os fatores de risco e os fatores de proteção presentes nesse contexto do desenvolvimento. Os fatores de risco são variáveis que tendem a aumentar a probabilidade de um indivíduo apresentar problemas físicos, sociais e emocionais, configuração que estará associada ao grau de ocorrência, intensidade, frequência, duração, severidade e percepção deste impacto para a pessoa em desenvolvimento (Poletto \& Koller, 2008; Yunes, 2003). Um estudo de Chiapetti (2003) com um grupo de pré-adolescentes constatou que comportamentos de risco como uso de drogas, sexualidade inapropriada, agressividade exacerbada e inadaptação social estavam associados negativamente aos aspectos provenientes do contexto familiar, sendo que tanto a comunidade quanto a escola se apresentaram como ambientes protetores em relação aos riscos analisados.

Entre os diversos fatores que podem atuar como de risco no desenvolvimento dos jovens, de forma a torná-los vulneráveis, Sapienza e Pedromônico (2005) destacam alguns riscos psicossociais, como a desnutrição, o atraso no desenvolvimento, os conflitos familiares, o desemprego e as dificuldades de acesso à saúde, ao lazer e à educação. Entre esses riscos destaca-se o fracasso escolar, que, juntamente com fatores associados como o desinteresse, a violência, a alfabetização deficitária e a falta de qualidade no ensino, é um dos problemas do processo de educação (Mazer, Belo, \& Mazon, 2009; Njaine \& Minayo, 2003).

No Brasil, diversos estudos evidenciam que o fracasso escolar está mais presente na vida dos adolescentes em escolas públicas. Para Tacca e Branco (2008), esta vivência pode gerar não apenas sentimentos negativos como, por exemplo, baixa autoestima, mas também problemas nas habilidades sociais, na aceitação pelos pares e na família, além de problemas comportamentais e emocionais. Leon e Menezes-Filho (2002) ressaltaram que, do ponto de vista individual, a escolha do nível educacional e o desempenho na escola podem ser influenciados por diversos fatores: as condições socioeconômicas do estudante, a compatibilidade do estudo com a inserção no mercado de trabalho, as condições econômicas e sociais da região onde se vive, as características pessoais, entre outras. Esses fatores, quando negativos, tendem a influenciar na manutenção do ciclo intergeracional de pobreza e podem dificultar os processos de aprendizagem (Black, Grenard, Sussmane, \& Rohrbach, 2010; Mazer e cols., 2009; Medeiros, Loureiro, Linhares, \& Marturano, 2000; Njaine \& Minayo, 2003; Sapienza \& Pedromônico, 2005) e a permanência do jovem na escola (Mazer e cols., 2009). Nesye sentido é fundamental a intervenção no espaço escolar.

A presença de fatores de proteção pode contribuir para amenizar o impacto dos riscos e promover estratégias de prevenção. Entre os fatores de proteção no contexto escolar - como cuidado, apoio, confiança, respeito pela adversidade e estrutura adequada - destaca-se a importância das relações com os professores e com os pares. A partir do estabelecimento de uma relação pautada pelo afeto confiança, é possível estimular uma escolarização positiva. Neste sentido, uma escola dotada de estruturas sociais adequadas para uma educação de qualidade poderá se configurar como espaço protetor, promovendo a autoestima e a autoeficácia e estimulando a construção de habilidades sociais, de aprendizagem, de afetividade e de relações e experiências positivas na escola (Amparo e cols., 2008; Bronfenbrenner, 2011; Camargo \& Libório, 2010; Dell'Aglio, 2010; Poletto \& Koller, 2008; Seligman \& Csikszentmihalyi, 2000).

Um estudo de Costa e Guedes (2009) que buscou compreender a percepção de alunos pertencentes à rede pública do Rio de Janeiro sobre suas aspirações e perspectivas de futuro apontou que o efeito-escola, a partir da criação de novos valores e hábitos, estaria relacionado com o aumento das expectativas de estudo, com a escolha da profissão e 
com as percepções mais positivas sobre seu futuro. As experiências escolares seriam promotoras de fatores de proteção decorrentes de um clima institucional de pertencimento e propício à aprendizagem, em que a perspectiva de um futuro melhor estaria vinculada a uma maior expectativa de estudo.

Mesmo estando associadas às vivências e experiências do contexto escolar, as expectativas de futuro, nas percepções dos jovens, também estão relacionadas com os seus esforços. Pesquisas apontam que o sucesso escolar se apresenta como um esforço individual que no decorrer do processo ensino-aprendizagem tende a ser determinante de maiores expectativas de estudo (Oliveira, Sá, Fischer, Martins, \& Teixeira, 2001). Um estudo de Amparo e cols. (2008) verificou que, apesar da percepção positiva de $73 \%$ dos jovens acerca da possibilidade de entrar para a universidade, $90,4 \%$ apontaram os seus esforços como determinantes para sua expectativa de futuro acadêmico; no entanto, Leon e Menezes-Filho (2002) destacaram, ainda, as questões socioeconômicas como principal determinante para o sucesso na escola. Com o passar das séries, estas questões apresentam-se como um "gargalo" para as expectativas de entrar numa universidade, o que demonstra que este sucesso não está associado apenas ao esforço individual, mas também às desigualdades sociais que passam a interferir, diminuindo as chances e crenças numa realização social e profissional.

Isto mostra a importância do contexto escolar para a construção da identidade, a formulação de planos e maiores expectativas de futuro. Compreender a escola como contexto de desenvolvimento, identificando os fatores de risco e os fatores de proteção, torna-se essencial para repensar as formas e estratégias que contribuem para o sucesso escolar e profissional, responsabilizando o jovem, a família e o conjunto social e a instituição escolar pela formação dos sujeitos sociais (Liebesny \& Ozella, 2002).

Assim, a função social da escola é fornecer ao indivíduo recursos para a sobrevivência em um mundo complexo e dinâmico, possibilitar sua comunicação com o mundo externo e garantir a transferência e a construção do conhecimento cultural e social, entendendo os papéis que emanam de cada relação e de cada contexto social e sua importância na construção de novos valores e hábitos (Bronfenbrenner, 1979/1996; 2011; Costa \& Guedes, 2009).

\section{Situação educacional de jovens no Brasil e no Pará}

Para Corbucci, Cassiolato, Codes e Chaves (2009), no Brasil há insuficiência de condições de acesso e de permanência na Educação Infantil e no Ensino Fundamental, o que resulta em sucessivas reprovações e evasão escolar, gerando uma elevada defasagem educacional. De acordo com esses autores, apesar do aumento das taxas de jovens brasileiros que concluem o Ensino Fundamental, os problemas de desempenho permanecem, com altos índices de reprovação e elevada distorção na relação idade-série. De acordo com o IBGE (2013), a reprovação escolar no Brasil passou de 12,1\% em 2007 para 10,3\% em 2010, enquanto a distorção idade/série passou de 44,9\% em 2006 para 37,8\% em 2010. A taxa de analfabetismo também vem caindo no Brasil: em 1992 era de 16,44\%, em 2005, de 10,5\%, em 2011 caiu para 7,9\%, demonstrando que cada vez mais os jovens participam da escola, embora ainda existam desafios nessa área.

Além disso, o Brasil caracteriza-se por uma intensa desigualdade regional, que se revela em diversos indicadores sociais, entre eles os educacionais. Assim, no estado do Pará, que está localizado na Região Norte, a segunda mais pobre do país, na Amazônia Brasileira, onde se realizou este estudo, a taxa de analfabetos chega a $11,23 \%$, enquanto nos estados da Região Sudeste, como Rio de Janeiro e São Paulo, esta taxa é de 4,0\%. No Pará, os dados do IBGE (2013) apontam que em 2010 as taxas de reprovação chegaram a $12,1 \%$ no Ensino Fundamental e $11,9 \%$ no Ensino Médio. No que se refere à porcentagem de abandono, esta taxa foi de 7\% no Ensino Fundamental e 19,2\% no Ensino Médio, no ano de 2010. Em São Paulo, por exemplo, as taxas de reprovação e abandono são respectivamente de 12, $6 \%$ e $4,5 \%$. Tais dados revelam ainda os imensos desafios educacionais para as regiões mais pobres do país.

Considerando esses dados e as implicações teóricas entre estas variáveis, este estudo teve como objetivo investigar as relações entre reprovação escolar eas percepções quanto à escola e às expectativas de futuro entre jovens paraenses. Parte-se de duas hipóteses: 1- a experiência de reprovação está relacionada a uma menor expectativa de futuro acadêmico; e 2- a expectativa de futuro acadêmico está relacionada com uma percepção mais positiva da escola.

\section{Método}

\section{Participantes}

Participaram 610 jovens, com idades entre 13 e 24 anos $(m=16,56 ; d p=2,37)$, dos quais $61,6 \%$ eram meninas e $38,4 \%$ eram meninos, todos estudantes de escolas da rede pública do município de Belém do Pará. Destes, 30,4\% estavam cursando entre a sétima e oitava séries do Ensino Fundamental e $69,6 \%$ estavam cursando o Ensino Médio. Os dados deste estudo transversal procedem do banco de dados da Pesquisa "Entre Risco e Proteção: o Ser Jovem em Belém do Pará" (Silva, 2013) do Instituto de Ciências da Educação, da Universidade Federal do Pará (ICED/UFPA).

\section{Instrumentos}

Foi utilizado um questionário para o levantamento dos fatores de risco e proteção, produzido pela pesquisa nacional sobre a Juventude Brasileira (Versão Fase II, Dell'Aglio, Koller, Cerqueira-Santos, \& Colaço, 2011). O instrumento contém 77 questões de múltipla escolha, que investigam as- 
pectos referentes à caracterização biosociodemográfica dos participantes e variáveis relacionadas aos aspectos educação, saúde, trabalho, violência, lazer e rede de apoio. Para este estudo foram utilizadas as questões 16 (reprovação), 17 (expulsão), 18 (Escala de Relações com a Escola, com sete itens sobre as percepções quanto à escola, envolvendo professores, técnicos e colegas, em escala Likert), e a questão 76 (sobre expectativas para o futuro). A questão 76 inclui nove itens, baseados na escala do tipo Likert construída por Günter e Günter (1998), em que o participante escolhe quais as chances que acredita ter de concluir o Ensino Médio, ter casa própria, constituir nova família, entre outros. Para este estudo foram analisadas apenas as questões referentes à perspectiva de concluir o Ensino Médio e entrar na universidade.

\section{Procedimentos e considerações éticas}

O projeto de pesquisa foi aprovado pelo Comitê de Ética em Pesquisa com Seres Humanos da UFPA, sob o protocolo 084/09 CEP_ICS/UFPA. A amostra foi composta de forma aleatória, por conglomerados, através do sorteio de dez escolas públicas do município de Belém. Também foram sorteadas as turmas participantes de cada escola. Foi solicitada aos pais a assinatura do Termo de Consentimento Livre e Esclarecido, em respeitos às normas estabelecidas pela Resolução no 196/96 do CNS (Conselho Nacional de Saúde) referentes a pesquisas envolvendo seres humanos. Os jovens também deram seu assentimento para a realização da pesquisa. $O$ instrumento foi aplicado coletivamente em sala de aula, por uma equipe treinada, durando aproximadamente uma hora. Foram realizadas análises descritivas e inferenciais, utilizando-se o Teste $t$ ou Qui-quadrado para verificar diferenças por sexo, e o Teste de Correlação de Pearson, para verificar correlações entre as variáveis investigadas.

\section{Resultados}

Foram analisadas as variáveis reprovação escolar, percepções quanto à escola e expectativas de futuro que estavam relacionadas à educação de 610 jovens paraenses. Em relação às experiências no contexto escolar, os dados indicaram que 46,9\% dos jovens já haviam tido reprovação escolar e 3,9\% já haviam sido expulsos da escola. Na tabela 1 são apresentados os resultados das análises da reprovação escolar por sexo. O teste de Qui-quadrado não indicou diferença significativa entre meninos e meninas, quanto aos percentuais de reprovação e expulsão escolar.

Tabela 1. Frequência e Percentuais de Reprovação e Expulsão Escolar por Sexo.

\begin{tabular}{llll}
\hline & Meninas & Meninos & Total \\
\hline Reprovação & $147(44,5 \%)$ & $105(50,2 \%)$ & $252(46,8 \%)$ \\
Expulsão & $12(3,7 \%)$ & $9(4,4 \%)$ & $21(3,9 \%)$ \\
\hline
\end{tabular}

Na tabela 2 são apresentadas as médias encontradas em cada item da Escala de Relações com a Escola e no total da escala. Observa-se que os itens "sentir-se bem na escola" e "gosto de ir para a escola" apresentaram as médias mais altas, enquanto "confiar nos colegas" apresentou a média mais baixa.

Tabela 2. Médias Quanto às Percepções sobre a Escola, por Sexo

\begin{tabular}{llll}
\hline Itens & Meninos & Meninas & Total \\
Eu me sinto & 3,96 & 4,15 & 4,08 \\
bem na escola & $(d p=1,13)$ & $(d p=1,19)$ & $(d p=1,16)$ \\
Gosto de ir para & 4,10 & 4,20 & 4,18 \\
a escola & $(d p=1,11)$ & $(d p=1,14)$ & $(d p=1,11)$ \\
Gosto da & 3,47 & 3,44 & 3,49 \\
maioria & $(d p=1,26)$ & $(d p=1,31)$ & $(d p=1,28)$ \\
dos meus & & & \\
professores & & & \\
Quero continuar & 3,92 & 3,68 & 3,81 \\
nessa escola & $(d p=1,44)$ & $(d p=1,56)$ & $(d p=1,50)$ \\
Posso contar & 3,64 & 3,46 & 3,56 \\
com meus & $(d p=1,30)$ & $(d p=1,29)$ & $(d p=1,29)$ \\
professores & & & \\
Posso contar & 3,48 & 3,61 & 3,57 \\
com técnicos da & $(d p=1,42)$ & $(d p=1,35)$ & $(d p=1,38)$ \\
escola & & & \\
Confio nos & 3,19 & 3,02 & 3,12 \\
colegas da & $(d p=1,33)$ & $(d p=1,38)$ & $(d p=1,36)$ \\
escola & & & $(d p=5.98)$ \\
Total na escala & 25,78 & 25,72 & $(d p=6,45)$ \\
& $(d p=5,47)$ & & \\
\hline
\end{tabular}

Na Tabela 3 são apresentadas, por sexo, as médias relativas às expectativas de futuro no tocante a concluir o Ensino Médio ou entrar para a universidade. Pode-se observar diferença significativa entre os sexos apenas na variável "expectativa de entrar na universidade", que apresentou média mais alta entre as meninas.

$\mathrm{Na}$ Tabela 4 são apresentadas as correlações entre as variáveis idade, série, quantidade de reprovações, percepção sobre a escola, expectativas de terminar o Ensino Médio e entrar numa universidade.

Foram observadas correlações significativas entre algumas variáveis e a idade, sendo que quanto maior a idade maior foi a vivência de reprovações e menor a expectativa de entrar na universidade. Também foram observadas correlações significativas, embora de fraca magnitude, entre a percepção quanto à escola e as expectativas de concluir o Ensino Médio e entrar na universidade. Além disso, foi encontrada uma baixa correlação negativa entre o número de repetências e a perspectiva de concluir o Ensino Médio, indicando que quanto mais vezes o aluno é reprovado menos ele tem expectativas de concluir o Ensino Médio. 
Tabela 3. Médias nas expectativas de futuro, por sexo.

\begin{tabular}{lllll}
\hline Expectativa & Meninos & Meninas & $t$ & $p$ \\
\hline Concluir o Ensino Médio & 4,19 & 4,33 & 1,63 & 0,10 \\
& $(d p=0,95)$ & $(d p=0,87)$ & & $<0,001$ \\
Entrar na universidade & 3,88 & 4,18 & 3,21 & $(d p=0,96)$ \\
\hline
\end{tabular}

Tabela 4. Correlações entre as Variáveis Investigadas.

\begin{tabular}{l|lllll}
\hline Variáveis & 2 & 3 & 4 & 5 & 6 \\
\hline 1. Idade &, $483^{* *}$ &, $316^{* *}$ &, 059 &, 040 &,$- 106^{*}$ \\
2. Série & 1 &, 080 &,- 046 &, $165^{* *}$ &,- 021 \\
3. Quantas vezes repetiu & & 1 &, 001 &,$- 163^{*}$ &,- 166 \\
4. Percepções quanto à Escola & & & 1 &, $201^{* *}$ &, $201^{* *}$ \\
5. Concluir o Ensino Médio & & & & 1 &, $557^{* *}$ \\
6. Entrar na Universidade & & & & & 1 \\
\hline
\end{tabular}

Nota: * $p<0,05 ;{ }^{* *} p<0,01$

\section{Discussão}

Os resultados deste estudo possibilitaram a compreensão das relações entre reprovação escolar, percepções quanto à escola e expectativas de futuro entre jovens. No que se refere às experiências de reprovação na vida dos jovens paraenses, pode-se observar que quase cinquenta por cento deles já tiveram reprovação em algum momento de sua vida, indicando uma diferença entre as taxas de reprovações individuais e as taxas brutas de reprovação aferidas pelo IBGE (2013). Leon e Menezes-Filho (2003) afirmam que a reprovação escolar é um dos principais problemas do sistema educacional, podendo trazer consequências negativas para a permanência na escola.

Por outro lado, os resultados deste estudo não apontaram relações entre o número de reprovações e as percepções quanto à escola. Apenas foi observada uma baixa correlação negativa com a perspectiva de concluir o Ensino Médio, sendo que quanto mais vezes o aluno tivesse sido reprovado, ele acreditava ser possível concluir seus estudos. Leon e Menezes-Filho (2003) constataram que o atraso escolar está associado a maior chance de abandonar os estudos imediatamente ou quando concluem os ciclos escolares. Assim, alunos com históricos de reprovação tendem a evadir-se após o término do Ensino Fundamental e do Ensino Médio, sendo esta situação denominada pelos autores de "efeito diploma". O fato de não ter sido observado efeito das reprovações sobre a percepção da escola pode ser compreendido a partir do significado que a escola tem para os jovens de classes populares. Apesar da repetência, o fato de os alunos permanecerem conectados ao espaço escolar pode ser entendido de forma positiva e estar relacionado a questões sociais. De acordo com Marques, Dell'Aglio e Sarriera (2009), para jovens de menor renda, o acesso a atividades que envolvem maior poder aquisitivo se torna difícil, sendo a escola um local mais valorizado por possibilitar acesso a recursos que não teriam em suas residências.

Diversos estudos destacam os determinantes da reprovação no ensino, tais como renda e idade, que resultam no atraso escolar e em sucessivas evasões (Corbucci e cols., 2009; Leon \& Menezes-Filho, 2003; MEC, 2005), constituindo-se a reprovação escolar como fator de risco para o desenvolvimento. Para Luszczynska, Gutiérrez-Donã e Schwarzer (2005), as emoções negativas que podem ser desencadeadas pela reprovação escolar podem se associar à presença de um baixo senso de autoeficácia, visto que as crenças de autoeficácia compõem os mecanismos de motivação e de participação dos alunos.

Também foi observada uma diferença significativa entre os sexos na variável "expectativa de entrar na univer- 
sidade", que apresentou média mais alta entre as meninas. Tal resultado indica dificuldades dos meninos em continuar os estudos, devidas às condições socioeconômicas. Um estudos do Instituto de Pesquisa Econômica Aplicada (IPEA) sobre as condições educacionais dos jovens brasileiros constatou que as mulheres têm se destacado no acesso à educação escolar (Corbucci e cols., 2009). Em 2007, a taxa de frequência líquida no Ensino Médio atingia 53,8\% entre as mulheres, aumentando as defasagens educacionais no sexo masculino, provenientes da dificuldade de conciliar os estudos com alguma atividade profissional, ou de problemas que extrapolam o âmbito escolar, como a violência e falta de renda. Os auores destacaram a necessidade de estudos mais detalhados sobre esta variável (Corbucci e cols., 2009; Leon \& Menezes-Filho, 2003).

O resultado referente às relações com a escola não demonstrou diferença significativa por sexo, mas foi observado que as médias dos itens "sentir-se bem na escola" e "gosto de ir para escola" foram mais altas que as médias de "confiar nos colegas". Tal resultado ressalta a necessidade de um olhar mais atento para as relações entre pares, visto que, dependendo da forma como se constroem estas relações, elas podem se configurar tanto como fator de proteção quanto fator de risco, pois essas relações podem influenciar os processos proximais e a formação de vínculos afetivos, aspectos tão importantes para a construção dos mecanismos de resiliência, para a permanência na escola e para o desenvolvimento humano (Amparo e cols., 2008; Bronfenbrenner, 2011; Poletto \& Koller, 2008). Esse resultado pode indicar a presença de dificuldades nas relações entre pares, as quais durante a adolescência podem se caracterizar também pela presença de violência e conflitos, como reflexo da presença da violência na sociedade (Santos, 2001); porém esse aspecto precisa ser melhor investigado em estudos que abordem de forma mais aprofundada as relações entre pares no ambiente escolar.

Também foi observada correlação significativa entre a percepção quanto à escola e às expectativas de terminar o Ensino Médio e entrar na universidade. Esse resultado indica que os alunos que percebem a escola de forma mais positiva, envolvendo colegas, professores e a escola como um todo, tendem a apresentar expectativas educacionais melhores para seu futuro. Pode-se destacar o papel do vínculo com professores e demais técnicos da escola na percepção em relação à escola. Para Black e cols. (2010), as relações informais e de orientação com professores e outros técnicos da escola favorecem o engajamento em atividades escolares. Para esses autores, as relações de orientações naturais que ocorrem ao longo do tempo entre um mentor (uma pessoa mais velha e com experiência) e um aprendiz, a partir da formação de vínculos afetivos fundados em confiança mútua e com a função de um recurso de apoio e orientação, podem favorecer seu desenvolvimento. Tais relações poderão também influenciar na manifestação de comportamentos pró-sociais, reduzindo o comportamento de risco.
A perspectiva de um futuro melhor mostrou-se relacionada com a percepção dos alunos sobre a escola, onde passam a construir projetos, planos e maiores expectativas escolares. Um estudo de Costa e Koslinski (2006) constatou que alunos com uma percepção mais positiva da escola possuíam uma vontade maior de dar continuidade aos estudos, ou seja, apresentavam maiores aspirações escolares e maior clareza quanto à definição de carreira. Por outro lado, os alunos que se encontravam desmotivados colocavam a culpa por tal desmotivação na escola e na educação, apresentando pouca aspiração escolar e perspectiva de carreira de menos prestígio social.

Neste sentido, a escola tem um importante papel no desenvolvimento dos jovens, não somente pelo desenvolvimento socioemocional e cognitivo, mas também pelo processo de socialização, da construção das identidades, dos planos e aspirações escolares e profissionais, atuando como fator de proteção. É de suma importância refletir tanto sobre as influências positivas da estrutura quanto dos professores e gestores, que podem oferecer uma rede de apoio que possibilite o enfrentamento das adversidades e perspectivas de um futuro melhor.

\section{Considerações finais}

Este estudo buscou investigar as relações entre reprovação escolar, percepções quanto à escola e expectativas de futuro entre jovens. Diante das hipóteses levantadas, foi possível constatar correlações significativas positivas entre a expectativa de futuro e uma percepção mais positiva da escola, em conformidade com a literatura. Os resultados contribuem para um melhor entendimento sobre a importância do microssistema escolar como contexto de desenvolvimento, pois a escola se constitui como uma importante rede de apoio para os jovens, uma vez que as relações, ações e atividades que acontecem dentro deste ambiente poderão se configurar como fator de proteção.

No que se refere à segunda hipótese levantada, a de que a experiência de reprovação estaria relacionada com uma menor expectativa de futuro, os resultados também foram significativos, apontando a experiência de reprovação como fator de risco, na medida em que diminui as expectativas de futuro acadêmico. Não obstante, os resultados demonstraram que a maioria dos alunos gosta de ir para escola e sente-se bem neste ambiente. Apesar de proporcionar experiências de fracasso, a escola ainda se apresenta como atrativa, com espaços de socialização e oportunidades (Amparo e cols., 2008; Poletto \& Koller, 2008). Tais dados podem indicar o forte papel da escola na agenda de sociabilidade dos jovens, como depositária de suas expectativas de futuro, o que reafirma a necessidade de investimento neste contexto e de revisão de sua proposta pedagógica, bem como dos modelos de gestão e de funcionamento.

Considera-se a necessidade de um olhar mais cuidadoso para as relações entre pares, pois as médias baixas em "confiar nos colegas" podem apresentar indícios da pre- 
sença de conflitos, violência na escola e de vitimização ou bullying. Tais comportamentos tornam-se fator de risco institucional e social, uma vez que podem produzir sérios danos psicológicos tanto para a vítima quanto para os agressores (Lisboa, Braga, \& Ebert, 2009).

Destaca-se que os resultados apresentados neste estudo referem-se a alunos matriculados na rede pública da Região Metropolitana de Belém do Pará, os quais forneceram importantes informações sobre a variável reprovação, relações com a escola e expectativa de futuro acadêmico, possibilitando a compreensão dos fatores de risco e dos fatores de proteção existentes nesse contexto. Por outro lado, esses resultados, embora se refiram a uma amostra aleatória e representativa, não podem ser generalizados para outros adolescentes, como, por exemplo, os matriculados em escolas particulares. São necessários novos estudos que investiguem, de forma longitudinal, esta realidade com adolescentes de diferentes contextos. Uma maior compreensão dos processos de escolarização e de suas falhas possibilita a construção de estratégias mais adequadas de educação, as quais são de grande importância para o desenvolvimento humano e para a sociedade, bem como para promoção de fatores de proteção que permitam a permanência e continuidade dos estudos.

\section{Referências}

Amparo, D. M., Galvão, A. C. T., Cardenas, C., \& Koller, S. H. (2008). A escola e as perspectivas educacionais de jovens em situação de risco. Revista de Psicologia Escolar e Educacional, 12, 69-88.

Bastos, J. C. (2005). Efetivação de escolhas profissionais de jovens oriundos do ensino público: Um olhar sobre suas trajetórias. Revista Brasileira de Orientação Profissional, 6(2), 31-43.

Beutel, A. M. (2000). The relationship between adolescent nonmarital childbearing and educational expectations: A cohort and period comparison. The Sociological Quarterly, 41(2), 297-314.

Black, D. S., Grenard, J. L., Sussman, S., \& Rohrbach, L. A. (2010). The influence of school-based natural mentoring relationships on school attachment and subsequent adolescent risk behaviors. Health Education Research, 25(5), 892-902.

Bronfenbrenner, U. (1996). A ecologia do desenvolvimento humano: experimentos naturais e planejados (M. A. V. Veronese, Trad). Porto Alegre: Artes Médicas. Trabalho original publicado em 1979

Bronfenbrenner, U. (2011). Bioecologia do desenvolvimento humano: Tornando os seres humanos mais humanos (A. C. Barreto, Trad). Porto Alegre: Artes Médicas.

Camargo, L. S., \& Libório, R. M. C. (2010). Do risco à proteção: O papel da escola na vida dos adolescentes do ensino médio. Em D. J. Silva, J. M. Lima \& P. C. Raboni (Orgs.), Pesquisa em educação escolar (pp.195-209). São Paulo: Cultura Acadêmica.
Chiapetti, N. (2003). Comportamento de risco em pré-adolescentes e contexto de convivência: influência do contexto escolar. Revista Electronica de Psicologia, 2.

Constituição da República Federativa do Brasil. (1988, 5 de outubro). Brasília: Senado Federal.

Corbucci, P. R., Cassiolato, M. M., Codes, A. L., \& Chaves, J. V. (2009). Situação educacional de jovens brasileiros. Em J. A. Castro, L. M. C. Aquino \& C. C. Andrade (Orgs.), Juventude e políticas sociais no Brasil (pp. 89-108). Brasília: IPEA.

Costa, M., \& Guedes, R. (2009). Expectativas de futuro como efeitoescola: Explorando possibilidades. São Paulo em Perspectiva, Fundação Seade, 23(1), 101-114.

Costa, M., \& Koslinski, M., C. (2006). Entre o mérito e a sorte: Escola, presente e futuro na visão de estudantes do Ensino Fundamental do Rio de Janeiro. Revista Brasileira de Educação, 11(31), 133201.

Dell'Aglio, D. D. (2010). A escola como contexto de desenvolvimento: Risco ou proteção? Em H. S. Antunes (Org.), Escola que protege: Dimensões de um trabalho em rede (pp. 109-119). Porto Alegre: Asterisco.

Dell'Aglio, D. D., Koller, S. H., Cerqueira-Santos, E., \& Colaço, V. F. R. (2011). Revisando o Questionário da Juventude Brasileira: Uma nova Proposta. Em D. Dell'Aglio \& S. Koller. (Eds.), Adolescência e Juventude: Vulnerabilidade e Contextos de Proteção (pp. 259270). São Paulo: Casa do Psicólogo.

Dessen, M. A., \& Polonia, A. C. (2007). A família e a escola como contextos de desenvolvimento humano. Paidéia, 17(36), 21-32.

Gomes, M. C., \& Horta, N. C. (2010). Promoção de saúde do adolescente em âmbito escolar. Revista de Atenção Primária à Saúde, 13, 486-499.

Günter, I. A., \& Günter, H. (1998). Brasílias pobres, Brasílias ricas: Perspectivas de futuro entre adolescentes. Psicologia: Reflexão e Crítica, 11, 191-207.

IBGE. (2013). Series estatísticas IBGE. Pesquisa nacional por amostra de domicílios 1992/2011. Recuperado: 19 jun 2013. Disponível: http://seriesestatisticas.ibge.gov.br/series

Leon, F. L. L., \& Menezes-Filho, N. A. (2003). Reprovação, avanço e evasão escolar no Brasil. Pesquisa e Planejamento Econômico, PPE, 32, 417-451.

Liebesny, B., \& Ozella, S. (2002). Projeto de vida na promoção de saúde. Em M. L. J. Contini, S. H. Koller \& M. N. S. Barros (Orgs.), Adolescência e psicologia: Concepções, práticas e reflexões críticas (pp.62-70). Rio de Janeiro: Conselho Federal de Psicologia. 
Lisboa, C., Braga, L. L., \& Ebert, G. (2009). O fenômeno bullying ou vitimização entre pares na atualidade: definições, formas de manifestação e possibilidades de intervenções. Contextos Clínicos, 2(1), 59-71.

Luszczynska, A., Gutierrez-Doña, B., \& Schwarzer, R. (2005). General self-efficacy in various domains of human functioning: Evidence from five countries. Psychology Press, 40(2), 80-89.

Marques, L. F., Dell'Aglio, D. D., \& Sarriera, J. S. (2009). O tempo livre na juventude brasileira. Em R. M. C. Libório \& S. H. Koller (Orgs.), Adolescência e juventude: Risco e proteção na realidade brasileira (pp. 79-106). São Paulo: Casa do Psicólogo.

Mazer, S. M., Bello, A. C., \& Bazon, M. R. (2009). Dificuldades de aprendizagem: revisão de literatura sobre os fatores de riscos associados. Psicologia da Educação, 28, 7-21.

Medeiros, P. C., Loureiro, S. R., Linhares, M. B. M., \& Marturano, E. M. (2000). A auto-eficácia e os aspectos comportamentais de crianças com dificuldades de aprendizagem. Psicologia: Reflexão e Crítica, 13(3), 327-336.

Ministério da Educação (MEC). (2005). Secretaria de Educação Infantil e Fundamental. Fracasso escolar no Brasil: Políticas, programas e estratégias de prevenção ao fracasso escolar. Brasília: MEC.

Njaine, K., \& Minayo, M. C. S. (2003). Violência na escolar: identificando pistas para a prevenção. Interface Comunicação, Saúde e Educação, 7(13), 119-134.

Oliveira, D. C., Sá, C. P., Fischer, F. M., Martins, I. S., \& Teixeira, L. R. (2001). Futuro e liberdade: $O$ trabalho e a instituição escolar nas representações sociais de adolescentes. Estudos de Psicologia, 6, 245- 258.
Poletto, M., \& Koller, S. H. (2008). Contextos ecológicos: Promotores de resiliência, fatores de risco e proteção. Estudos de Psicologia, 25(3), 405-416.

Santos, J. V. T. (2001). A violência da escola: Conflitualidade sócias e ações civilizatórias. Educação e Pesquisa, 27(1), 105-122.

Sapienza, G., \& Pedromônico, M. R. M. (2005). Risco, proteção e resiliência no desenvolvimento da criança e do adolescente. Psicologia em Estudo, 10(2), 209-216.

Seligman, M. E. P., \& Csikszentmihlyi, M. (2000). Positive psychology: An introduction. American Psychologist, 55(1), 05-14.

Silva, L. I. C. (2013). Entre risco e proteção: o ser jovem em Belém do Pará. Relatório final de pesquisa. Belém: ICED/UFPA.

Tacca, M. C. V. R., \& Branco, A. U. (2008). Processos de significação na relação professor aluno: Uma perspectiva sociocultural construtivista. Estudos de Psicologia, 13(1), 39- 48.

Tavares, J. (2002). A resiliência na sociedade emergente. Em J. Tavares (Org.), Resiliência e Educação (pp. 44-75). São Paulo: Cortez.

UNESCO. (1998). Educação um tesouro a descobrir. Relatório para UNESCO da Comissão Internacional sobre Educação para o Século XXI. Brasília.

Yunes, M. A. M. (2003). Psicologia positiva e resiliência: Foco no indivíduo e na família. Psicologia em Estudos, 8 (num. esp.), 7584.

Yunes, M. A. M., \& Szymansky, H. (2001). Resiliência: Noção, conceitos afins e considerações críticas. Em J. Tavares (Org.), Resiliência e Educação (pp.13-42). São Paulo: Cortez.

\section{Sobre os autores}

Tatiene Germano Reis Nunes (tatienegermano@gmail.com)

Universidade Federal do Pará

Fernando Augusto Ramos Pontes (farp1304@gmail.com)

Universidade Federal do Pará

Lucia Isabel da Conceição Silva (luciibel@yahoo.com.br)

Universidade Federal do Pará

Débora Dalbosco Dell'Aglio (dalbosco@cpovo.net)

Universidade Federal Rio Grande do Sul 